\title{
Mechanical properties of lipid bilayers
}

\section{INTRODUCTION}

Everyone agrees that the bending modulus (also known as $\mathrm{K}_{\mathrm{c}}$ or $\mathrm{K}$ ) is an important property of biomembranes, as it quantifies the flexibility that is important to cell functions (Nagle, 2013). For many years, we have used the traditional Canham-Helfrich model for fluctuations to analyse diffuse X-ray scattering in order to obtain values for the bending modulus $\mathrm{K}_{\mathrm{C}}$ (Liu \& Nagle, 2004). Theory and molecular dynamics simulations have suggested that the next level of refinement to describe membrane fluctuations should extend this model to include molecular tilting (May et al., 2007). This extension is widely considered to be important for understanding membrane fusion (Ryham et al., 2016). Of the two versions of an extended model, the Brown model (Watson et al., 2011) has been shown to provide the more accurate description of the hydrocarbon chains as determined by simulations (Kopelevich \& Nagle, 2015).

\section{EXPERIMENTAL}

Our primary experimental technique is X-ray scattering from stacks of fully hydrated lipid bilayers. In addition to the usual quasi-Bragg peaks, these samples exhibit extensive diffuse scattering that contains more information than the peaks. In particular, we have been obtaining the bending modulus $\mathrm{K}_{\mathrm{c}}$ from diffuse scattering, which is one focus of this talk. Quantities also obtained that were not focused on are the electron density profile and the bulk $B$ modulus that is a measure of interactions between neighbouring bilayers in the stack. Recently, we have learned how to include molecular tilt in our analysis of X-ray data. We found that the X-ray data are fit better for the tilt-dependent model, and we have reported the first experimental value of the tilt modulus $\mathrm{K}_{\theta}$ for dioleoylphosphatidylcholine lipid bilayers (Jablin et al., 2014). This has required considerable theoretical, analytical and software development (Jablin, 2015). Re-analysis that includes the tilt modulus is now being performed on extensive earlier $\mathrm{X}$-ray scattering data collected in my laboratory.

\section{RESULTS AND DISCUSSION}

Preliminary results for the tilt modulus of dimyristoylphosphatidylcholine (DMPC) were shown. It is noteworthy that two recent atomistic simulations (Venable et al., 2015; Wang \& Deserno, 2016) each performed at only one temperature, agree well with our experimental results. However, my most striking result, not yet investigated by simulations, is the rapid decrease in $\mathrm{K}_{\theta}$ as the main transition in DMPC is approached by lowering the temperature within the fluid phase. This provides a new perspective for the DMPC main phase transition. In critical phenomena, the vanishing of the modulus corresponding to an order parameter results in spontaneous symmetry breaking of that order parameter. It is well known that there is spontaneous tilt in the gel phase. Although the main transition is into the ripple phase that is complicated by ripple symmetry breaking, recent structural work (Akabori \& Nagle, 2015) shows that the ripple phase also 
has spontaneous tilt (along with other interesting features not comprehended by existing theory). This supports the previous interpretation that there is pre-critical behaviour above the transition, as originally evidenced by the accelerating decrease in volume on approach to the transition (Nagle \& Wilkinson, 1978). However, whilst tilt acts like an order parameter with spontaneous symmetry breaking at the transition, there is clearly much more going on, and area/lipid is probably the most important order parameter (Nagle, 1986) and a full theory should probably also include the spontaneous symmetry breaking of rippling in the ripple phase.

The new tilt-dependent analysis continues to find that $\mathrm{K}_{\mathrm{c}}$ also decreases as the main transition is approached from the fluid phase (Chu et al., 2005). The decrease now begins at a lower temperature $\left(27^{\circ} \mathrm{C}\right)$ than the previous one $\left(30^{\circ} \mathrm{C}\right)$, above which $\mathrm{K}_{\mathrm{c}}$ decreases with increasing temperature. The new maximum agrees better with the onset of the anomalous swelling regime.

The older tilt-independent analysis essentially assumed that the tilt modulus was infinite. Allowing a finite tilt modulus in the analysis of a data set increases the obtained value of $\mathrm{K}_{\mathrm{c}}$.
Values of the bending modulus $\mathrm{K}_{\mathrm{c}}$ from the new tilt-dependent analysis were compared to those obtained by the classical methods that use giant unilamellar vesicles. Compared to what has been discussed previously (Nagle, 2013) this reduces the differences between the $\mathrm{X}$-ray values of $\mathrm{K}_{\mathrm{C}}$ and the generally larger values obtained by other experimental methods that sense membrane flexibility on much larger length scales than the X-ray method and that are, therefore, insensitive to tilt (Nagle et al., 2015). Nevertheless, there are still outstanding discrepancies in the values of the bending modulus that might have been due to the effect of sugar used at different concentrations in the classical experiments. However, we have been unable to find the effect of sugars in our experiments (Nagle et al., 2016).

\section{ACKNOWLEDGEMENT}

I thank the 4th European Joint Theoretical/Experimental Meeting on Membranes (7-9 September, 2016, in Bratislava, Slovakia) and its organisers for sponsoring my travel.

\section{References}

[1] Akabori K, Nagle JF. Structure of the DMPC lipid bilayer ripple phase. Soft Matter 2015; 11:918-926.

[2] Chu N, Kucerka N, Liu YF, Tristram-Nagle S, Nagle JF. Anomalous swelling of lipid bilayer stacks is caused by softening of the bending modulus. Phys Rev E. 2005; 71:041904(1-8).

[3] Jablin MS, Akabori K, Nagle JF. Experimental Support for TiltDependent Theory of Biomembrane Mechanics. Phys Rev Lett. 2014;113: 248102 (1-5).

[4] Jablin, M. S., Tilt-Dependent Analysis of Diffuse X-ray Scattering from Oriented Stacks of fluid Phase Lipid Bilayers. Thesis: http:// lipid.phys.cmu.edu, 2015; pp. 1-257.

[5] Kopelevich DI, Nagle JF. Correlation between length and tilt of lipid tails. J Chem Phys. 2015; 143:154702(1-8).

[6] Liu YF, Nagle JF. Diffuse scattering provides material parameters and electron density profiles of biomembranes. Phys Rev E. 2004;69:040901(1-4).

[7] May ER, Narang A, Kopelevich DI. Role of molecular tilt in thermal fluctuations of lipid membranes. Phys Rev E. 2007;76:021913(1-6).

[8] Nagle JF. Introductory Lecture: Basic quantities in model biomembranes. Faraday Discuss. 2013;161:11-29.

[9] Nagle JF, Wilkinson DA. Lecithin Bilayers - Density-Measurements and Molecular-Interactions. Biophys J. 1978;23:159-175.
[10] Nagle JF. Theory of Lipid Monolayer and Bilayer Chain-Melting Phase-Transitions. Faraday Discuss. 1986;81:151-162.

[11] Nagle JF, Jablin MS, Tristram-Nagle S, Akabori K. What are the true values of the bending modulus of simple lipid bilayers? Chem Phys Lipids. 2015;185:3-10.

[12] Nagle JF, Jablin MS, Tristram-Nagle S. Sugar does not affect the bending and tilt moduli of simple lipid bilayers. Chem Phys Lipids. 2016;196:76-80.

[13] Ryham RJ, Klotz TS, Yao LH, Cohen F S. Calculating Transition Energy Barriers and Characterizing Activation States for Steps of Fusion. Biophys J. 2016;110:1110-1124.

[14] Venable RM, Brown FLH, Pastor RW. Mechanical properties of lipid bilayers from molecular dynamics simulation. Chem Phys Lipids. 2015;192:60-67.

[15] Wang X, Deserno M. Determining the Lipid Tilt Modulus by Simulating Membrane Buckles. J Phys Chem B. 2016;120:60616073.

[16] Watson MC, Penev ES, Welch PM, Brown FLH. Thermal fluctuations in shape, thickness, and molecular orientation in lipid bilayers. J Chem Phys. 2011;135:(1-22). 